\title{
Contraintes de taille dans les mots composés : quand la phonologie entre en concurrence avec les contraintes morphologiques ${ }^{1}$
}

\author{
Villoing, Florence \\ Université Paris 8 Saint-Denis \& Cnrs Umr 7023 SFL \\ Florence.Villoing@univ-paris8.fr
}

\section{Introduction}

Cet article porte sur les contraintes phonologiques qui pèsent sur la formation des mots construits. En étudiant les données du français, Corbin (à paraître), Lignon (1999), Plénat (2000), Roché (1997), les premiers, ont montré que les exigences sémantiques et les exigences phonologiques peuvent entrer en conflit lors de la construction morphologique d'unités lexicales, et comment les unes cèdent sur les autres. En particulier, les travaux de Lignon (à paraître), Plénat et Roché ont montré que des contraintes de taille étaient à l'oeuvre dans plusieurs constructions morphologiques par suffixation (y compris dans des formations relevant de la morphologie périphérique comme les suffixations substitutives familières ou populaires), ou dans des procédés périphériques à la morphologie (oralisation des sigles, javanais). l'hypothèse peut être étendue à la composition morphologique. Ainsi, Villoing (2009), repris par Fradin (2009), a proposé que des contraintes de taille puissent être à l'oeuvre dans la formation des mots composés Verbe-Nom (désormais VN) (ouvre-boîte, lèche-vitrine, casse-pied); la démonstration s'appuyait sur l'observation que cette construction sélectionne très majoritairement des verbes monosyllabiques, excluant quasiment tout autre verbe de taille supérieure.

L'objectif de cet article est d'étendre la recherche à la taille du N des mots composés VN et à la taille des composés eux-mêmes. Cette recherche s'inscrit dans le cadre théorique de la morphologie lexématique qui, au contraire de la morphologie morphématique, envisage la formation des lexèmes au moyen de procédés relevant de fonctions s'appliquant aux lexèmes bases pour fournir des lexèmes plus complexes (pour une comparaison des deux modèles, voir, entre autres, Anderson 1992, Aronoff 1994, Fradin 2003). Dans ce cadre théorique, la composition est reconnue comme un procédé morphologique et non syntaxique. Les mots composés VN sont ainsi traités comme construits par une règle morphologique qui prend pour base deux lexèmes, un verbe et un nom, et s'applique simultanément à chacune des trois dimensions définitoires de ces lexèmes (phonologique, catégorielle et sémantique) pour construire un lexème plus complexe, le mot composé VN, catégorisé nom ou adjectif (voir Corbin 1992, Fradin 2009 et Villoing 2009, 2012 pour les détails).

La question qui nous occupe dans cet article est de déterminer si les contraintes phonologiques de taille que la règle fait peser sur la formation des mots composés VN ne porte que sur le verbe, comme le supposait Villoing (2009), ou s'imposent également au nom voire au mot composé dans son entier. En nous interrogeant sur les raisons qui poussent à contraindre la taille des lexèmes, nous tenterons d'élucider le lien entre contraintes de taille des composants des mots composés VN et nécessité de répondre à un idéal dissyllabique.

La première partie $(\S 2)$ s'attachera à présenter les propriétés phonologiques du mot prosodique optimal $\mathrm{du}$ français, en rappelant que les mots du français tendent vers un idéal dissyllabique. Elle établira également sur quels critères s'appuyer pour mener à bien un décompte syllabique. La seconde partie (§ 3) présentera un décompte syllabique des mots composés VN en fournissant d'abord les résultats qui concernent les verbes puis les noms. Ce calcul s'appuiera sur la capacité des voyelles et des consonnes à former de bonnes attaques et des consonnes à constituer de bonnes codas. Le décompte sera mené sur le corpus lexicographique recueilli dans Villoing (2002) qui rassemble les mots composés VN figurant dans plusieurs grands dictionnaires du français tels que le TLF, le Grand Robert ou le Grand Larousse ${ }^{2}$. Les parties suivantes s'interrogeront sur le fait que les mots composés VN répondent à un idéal dissyllabique 
(§ 4) et si ce n'est pas le cas, s'ils subissent ou non des contraintes de taille (§ 5). La conclusion s'ouvrira sur de nouvelles perspectives, théoriques et empiriques, en particulier la recherche de contraintes de taille jouant sur d'autres construits morphologiques tels que les converts $\mathrm{V}>\mathrm{N}$.

\section{Propriétés phonologiques du mot prosodique optimal en français}

\subsection{Idéal dissyllabique du mot en français}

Les contraintes de taille sont reconnues comme omniprésentes dans les langues du monde (cf. Plénat 2000). Au sein des contraintes prosodiques universelles (cf. les contraintes de fidélité - qui cherchent à maintenir l'identité phonologique de la base dans le dérivé - et les contraintes anti-marques - qui tendent à favoriser les configurations sonores les moins marquées du point de vue de l'articulation et/ou de la perception; voir Prince \& Smolensky 1993), les contraintes de taille appartiennent à la famille des contraintes anti-marques (au même titre, par exemple, que les contraintes dissimilatives qui tendent à éviter l'ajout de matériel phonologique identique à celui de la base). Les contraintes de taille stipulent que, dans les langues, la taille minimum des mots est, couramment de deux syllabes. Les travaux sur le français (cf. pour une synthèse, Plénat 2009) ont défendu l'hypothèse que les contraintes à l'œuvre font de deux syllabes un minimun et un maximum. En d'autres termes, en français, le mot prosodique minimal et optimal est de deux syllabes (cf. aussi Kilani-Schoch \& Dressler 1992). Cet idéal dissyllabique a été identifié dans l'oralisation des sigles (Plénat 1993, 1997b) et dans des modes de formation morphologiques relativement marginaux tels que les hypocoristiques (Plénat 1984, 1999) et la suffixation substitutive (Plénat 1997a) mais aussi dans des formations plus centrales de la morphologie comme la suffixation en -iser et -ifier en français (Lignon (à paraître)), les accourcissements (Plénat 2002, Plénat \& Roché 2003) ou les allongements (parfois appelés «suffixation décalée » ou « interfixation ») (Plénat 2005, Plénat \& Roché 2004, Roché 2002, 2003, 2009).

\subsection{Structure du dissyllabe}

Les contraintes de syllabation des mots du français sont identiques, qu'il s'agisse de lexèmes simples ou de lexèmes construits. En somme, « en français, pour qu'une séquence puisse être syllabée, il faut qu'elle comporte au moins une voyelle et que les séquences de consonnes qui entourent ces voyelles constituent des attaques et des codas possibles dans la langue. »(Plénat 1997b : 35). Les contraintes anti-marque jouent là aussi sur la forme que prennent les syllabes : elles tendent à favoriser des syllabes de type CV dont l'attaque comme la rime seraient simples (Plénat 2009). Comme un mot doit autant que possible commencer par une attaque, certains travaux ont montré qu'au besoin, une voyelle initiale peut ne pas entrer dans le décompte des syllabes (Plénat parle d' "extramétricité des voyelles initiales »). Dans ces cas là, les dissyllabes à initiale vocalique se comportent comme les monosyllabes.

\subsection{Choix du matériel segmental}

Les contraintes anti-marque postulent également une hiérarchie des attaques qui indiquent le degré d'aptitude des consonnes aux fonctions d'attaque et de coda. Ces hiérarchies jouent un rôle dans la syllabation du français (Plénat 1999). Elles sont liées à des facteurs phonologiques que l'on retrouve avec une certaine constance dans toutes les langues du monde. Pour reprendre les termes de Plénat (1999) : « Il est préférable qu'une attaque soit momentanée plutôt que continue et obstruante plutôt que sonante ; toutes choses égales par ailleurs, il vaut mieux qu'une coda soit une sonante plutôt qu'une obstruante et une continue plutôt qu'une momentanée.». Ainsi, les occlusives sont les plus aptes à remplir les fonctions d'attaque tandis que les consonnes telles que $/ \mathrm{r} /$ et $/ \mathrm{z} /$ le sont moins. 


\section{Contraintes de taille sur les composants}

La composition $\mathrm{VN}$, en tant que règle morphologique de formation de lexèmes, se définit par un ensemble de contraintes morphologiques qui définissent la forme phonologique de la base et du construit. Elle impose notamment la sélection d'un thème (ou radical) spécifique des lexèmes bases, en l'occurence un des thèmes du verbe et du nom du mot composé. Nous rendons compte ici de ces contraintes en nous inscrivant dans le cadre théorique des espaces thématiques proposé par Boyé \& Bonami (2003) pour le français, initialement destiné à l'étude des verbes, étendu depuis à celui des adjectifs (Bonami \& Boyé 2005) et des noms (Plénat 2008, Roché 2010). Selon cette optique, les lexèmes sont identifiés phonologiquement par un ensemble de thèmes (ou radicaux) liés entre eux au sein d'un espace thématique. Les verbes comprennent 12 thèmes qui servent à construire leur paradigme (ou 13 thèmes selon Bonami, Boyé, Kerleroux 2009), et les noms 2 thèmes. Villoing (2009) a montré que la composition $\mathrm{VN}$ sélectionne le thème 3 du verbe, celui de l'indicatif présent singulier et le thème 1 du nom, celui de la forme libre masculin.

La composition VN semble en outre imposer des contraintes phonologiques. En effet, l'étude du format syllabique des mots composés $\mathrm{VN}$ du français conduit à reconnaître que leurs composants sont soumis à des contraintes de taille. La démonstration s'appuie sur le calcul syllabique des verbes et des noms qui les composent révélant que les verbes sont très massivement monosyllabiques, et que les noms, quoi que moins contraints, le sont aussi préférentiellement.

\subsection{Contraintes de taille sur les verbes}

\subsubsection{Des verbes majoritairement monosyllabiques}

Les premiers travaux engagés sur les contraintes de taille dans les mots composés VN (Villoing 2009) ont montré que la règle sélectionne préférentiellement des verbes monosyllabiques. Ainsi, sur les 160 verbes du corpus exploités pour construire les mots composés VN, 128 comprennent une voyelle unique, ce qui représente $80 \%$ de l'ensemble des verbes (voir Tableau 1.) (citons à titre d'exemple, des verbes très présents dans le corpus tels que brise, casse, coupe, passe, porte, tire que l'on retrouve dans les exemples (1)).

brise-glace, casse-cou, coupe-gorge, passe-plat, porte-plume, tire-fesses

Cette très nette majorité peut encore augmenter si l'on prend en compte certains verbes dissyllabiques comprenant soit une syllabe susceptible de chuter à cause d'un schwa non réalisé, soit une syllabe initiale à mauvaise attaque (cf. § 2.3), conduisant ainsi à les recalculer comme monosyllabiques. Par exemple, l'initiale en / $\mathrm{r}$ / suivie d'un schwa de verbes a priori disyllabiques tels que rebrousse, relève, remonte, remue, repose dans les exemples (2) ne constitue certainement pas une syllabe, le schwa pouvant tomber en syllabe initiale de mot «lorsqu'il est précédé d'une consonne, sauf s'il est à la fois précédé et suivi d'une obstruante non-continue » (Dell $1973: 225$ ).

(à) rebrousse-poil, relève-moustache, remonte-pente, remue-ménage, repose-pied

Un calcul similaire pourrait être avancé pour les verbes dissyllabiques dont la première syllabe comprend une voyelle pouvant ne pas entrer dans le décompte des syllabes (ex. 3) (cf. § 2.2 ci-dessus qui expose l'hypothèse de Plénat selon laquelle les dissyllabes à initiale vocalique peuvent se comporter comme des monosyllabes).

abaisse-langue, arrête-boeuf, enfile-aiguille, étouffe-chrétien, épluche-légumes

A ce compte, le nombre de verbes dissyllabiques risque de se voir réduit à portion congrue. Le Tableau 1 montre que les verbes dissyllabiques qui apparaissent dans les mots composés VN du corpus, et dont la 
première syllabe contient une bonne attaque (cf. § 2.3) ne sont qu'au nombre de 8, ce qui ne représente que $5 \%$ des verbes.

\begin{tabular}{lcc}
\hline V d'un composé comportant : & Total (sur 160 Vb) & Pourcentage \\
\hline $\begin{array}{l}\text { - une syllabe } \\
\quad \text { deux syllabes dont une voyelle à }\end{array}$ & $\mathbf{1 2 8}$ & $\mathbf{8 0} \%$ \\
l'initiale & 19 & $11,87 \%$ \\
$\quad$ - deux syllabes dont initiale en /rə/ & 5 & $3,1 \%$ \\
$\quad-$ deux syllabes sans initiale en voy. ni & 8 & $5 \%$ \\
/ro/ & & \\
\hline
\end{tabular}

\section{Tableau 1. Taille des verbes des composés VN}

Conclure que seuls 5\% des verbes des mots composés VN sont dissyllabiques n'est cependant pas sérieux ; les propositions de recalculs syllabiques avancées ici doivent être pris avec précaution, pour au moins deux raisons. D'une part parce qu'aucune règle n'impose a priori une telle syllabation des mauvaises attaques puisqu'elle dépend du poids respectif des contraintes que la langue impose. Et d'autre part parce que le calcul syllabique du verbe ne peut se faire qu'au sein du mot composé dans son entier, conduisant éventuellement au résultat inverse (faisant d'un monosyllabe un dissyllabe). En effet, les schwas immédiatement précédés de deux consonnes appartenant au même mot ne tombent pas lorsque la syllabe suivante porte l'accent principal de mot composé. Ainsi le schwa situé en finale de polysyllabe « se maintient toujours dans les mots comme porte-plume, porte-voix, garde-meuble, ouvre-boite, où le second terme du composé n'a phonétiquement qu'une syllabe, tandis qu'il tombe facultativement dans des mots comme port(e)-drapeau, gard(e)-malade, gard(e)-barrière, ouvr(e)-bouteille.» (Dell 1973: 222-224). Ainsi, le maintien du schwa final de porte dans porte-plume conduirait à comptabiliser un verbe dissyllabique, tandis que la forte probabilité de la chute du schwa dans port(e)-drapeau entraînerait à ne comptabiliser pour le verbe qu'une syllabe. En outre, il faudrait prendre en compte la propriété qu'ont certaines consonnes finales à constituer des attaques de syllabes dégénérées, qui entreraient ainsi dans le décompte syllabique, et de ce fait, ajouteraient une syllabe incomplète (cf. Plénat 1998, 1999, 2002, 2009).

Cependant, la proportion des verbes monosyllabiques est suffisamment massive pour conclure que des contraintes de taille pèsent sur les verbes des mots composés VN.

\subsubsection{Taille des verbes dans le lexique général du français}

Pour être véritablement valide, ce résultat devrait tenir compte de la proportion, dans le lexique général $\mathrm{du}$ français, des verbes monosyllabiques relativement aux verbes plurisyllabiques, ce qui permettrait de mesurer si la représentation massive des verbes monosyllabiques dans les mots composés VN n'est pas tout simplement liée à leur caractère massif dans le lexique.

A l'issue de l'étude, il s'avère que notre résultat est non seulement fiable mais en plus particulièrement significatif. En effet, la proportion de $80 \%$ de thèmes verbaux monosyllabiques dans le corpus des VN est exceptionnellement élévée au regard de la proportion de ce type de thèmes dans le lexique général du français. Ainsi, en interrogeant un lexique représentatif du français tel que Lexique 3 (cf. New B., Pallier C., Ferrand L., Matos R. (2001), en ligne www://lexique.org), on observe que sur les 1088 formes verbales correspondant au thème $3 \mathrm{du}$ verbe (celui qui apparaît dans les formes du présent, indicatif, singulier), enregistrées dans la base, seules 202 sont monosyllabiques, soit 18,5\% (cf. Tableau 2.). En d'autres termes, la taille du thème 3 des verbes du français est loin d'être majoritairement monosyllabique, comme elle l'est dans les mots composés VN. En revanche, le tableau 2 montre 
clairement que le thème 3 des verbes du français est de préférence égal ou supérieur à un dissyllabe (52,6\% de verbes dissyllabiques et $23,8 \%$ de verbes trisyllabiques).

\begin{tabular}{lcc}
\hline Thème 3 du verbe : & Total (sur $1088 \mathrm{Vb})$ & Pourcentage \\
\hline - monosyllabique & 202 & $18,5 \%$ \\
- dissyllabique & $\mathbf{5 7 3}$ & $\mathbf{5 2 , 6 \%}$ \\
- trisyllabique & 259 & $23,8 \%$ \\
- quadrisyllabique & 49 & $4,5 \%$ \\
- cinq syllabes & 5 & $0,45 \%$
\end{tabular}

Tableau 2. : Taille du thème 3 des verbes du français

La comparaison de la taille des verbes dans les mots composés VN avec celle des verbes du lexique général du français révèle des tendances nettement distinctes. Elle conduit à conclure que l'apparition massive de verbes monosyllabiques dans ces mots composés n'est pas le reflet des propriétés des verbes du français, en général. Au contraire, elle montre sans ambiguïté qu'une contrainte de taille, ressortissant à la règle morphologique de formation de ces composés, pèse sur le $\mathrm{V}$ des mots composés VN.

\subsubsection{Taille des verbes les plus fréquents dans les VN}

Les chiffres présentés en 3.1.1. ne permettent cependant pas d'identifier les verbes les plus exploités dans le corpus, les verbes monosyllabiques pouvant être certes nombreux mais néanmoins formateurs de peu de mots composés. Cette information permettrait ainsi de reconnaître, au-delà de la diversité des verbes, si le choix préférentiel des locuteurs porte véritablement sur les verbes monosyllabiques. Le résultat de cette mesure quantitative devrait néanmoins être prise avec précaution parce qu'elle mêle des préférences de taille à des préférences sémantiques : si un verbe comme porter a été largement utilisé pour former les mots composés VN (porte-avion, porte-bagages, porte-bonheur, porte-couverts, porte-lance, portemonnaie, porte-plume, porte-voix), la raison tient probablement aussi à sa particulière souplesse sémantique (pour ne pas dire polysémie) et à sa pertinence pour dénoter ce que cette construction cherche à dénoter.

La place nous manque ici pour fournir de tels résultats mais une rapide attention au nombre de mots composés VN construits avec un verbe mono- ou dissyllabique met clairement au jour le fait que les verbes les plus exploités sont monosyllabiques. Ainsi, les radicaux tels que porte, garde, passe, tire, coupe, cache, chasse ou casse sont ceux qui ont permis de construire le plus de mots composés. En outre, aucun verbe dissyllabique ne figure parmi les verbes les plus fréquents (c'est-à-dire ceux qui apparaissent au minimum dans 20 mots composés $\mathrm{VN}$ du corpus) (cf. Tableau 3.).

\begin{tabular}{|l|l|l|l|l|}
\hline Verbe & $\begin{array}{l}\text { Nbr syll. } \\
\mathrm{V}\end{array}$ & $\begin{array}{l}\text { Nb de VN } \\
\text { constuits avec le } \\
\text { V }\end{array}$ & $\begin{array}{l}\% \text { (sur 1473 } \\
\text { VN) }\end{array}$ & Exemples \\
\hline porte & $\sigma$ & 291 & $19,7 \%$ & porte-serviettes, porte-bébé \\
\hline garde & $\sigma$ & 78 & $5,3 \%$ & garde-malade, garde-manger \\
\hline passe & $\sigma$ & 72 & $4,9 \%$ & passe-purée, passe-droit \\
\hline tire & $\sigma$ & 57 & $3,8 \%$ & tire-fesses, tire-lait \\
\hline coupe & $\sigma$ & 45 & $3 \%$ & coupe-vent, coupe-gorge \\
\hline cache & $\sigma$ & 39 & $2,64 \%$ & cache-misère, cache-pot \\
\hline chasse & $\sigma$ & 38 & $2,57 \%$ & chasse-mouches, chasse-neige \\
\hline casse & $\sigma$ & 35 & $2,37 \%$ & casse-cou, casse-noix \\
\hline couvre & $\sigma$ & 28 & $1,9 \%$ & couvre-feu, couvre-lit \\
\hline perce & $\sigma$ & 25 & $1,7 \%$ & perce-muraille, perce-oreille \\
\hline
\end{tabular}




\begin{tabular}{|l|l|l|l|l|}
\hline serre & $\sigma$ & 24 & $1,6 \%$ & serre-tête, serre-papiers \\
\hline brise & $\sigma$ & 22 & $1,5 \%$ & brise-glace, brise-cou \\
\hline monte & $\sigma$ & 20 & $1,35 \%$ & monte-livres, monte-charge \\
\hline
\end{tabular}

Tableau 3. : Verbes les plus fréquents dans les mots composés VN

Ce déséquilibre n'apparaît pas avec les verbes les moins exploités puisque, dans ce cas, sont aussi bien représentés les thèmes monosyllabiques que dissyllabiques (cf. Tableau 4). On peut cependant avancer l'hypothèse que les raisons de l'absence de productivité de ces verbes dans les mots composés VN sont certainement différentes : les verbes monosyllabiques ci-dessous semblent fort peu productifs en français contemporain (hormis cueille et prête, on ne trouve que relativement peu d'emplois des verbes dompte, ferre, foule, enfle, hume, mate sur la Toile). Tandis que les verbes dissyllabiques du Tableau 4 (en admettant qu'ils ne soient pas recomptables comme monosyllabes, hypothèse pourtant fort plausible, cf. 3.1.1.) en dehors peut-être de ratisse, sont d'un usage très courant (amuse, étouffe, épluche, écorche, rebrousse, remonte, copie). Ces observations nous conduisent à conclure que leur absence dans les mots composés $\mathrm{VN}$ n'est pas liée à leur faible fréquence en français mais plutôt à leur valeur sémantique, possiblement trop spécifique pour la composition en question.

\begin{tabular}{|l|l|l|l|l|}
\hline cueille & $\sigma$ & 1 & $0,06 \%$ & cueille-fruits \\
\hline dompte & $\sigma$ & 1 & $0,06 \%$ & dompte-venin \\
\hline enfle & $\sigma$ & 1 & $0,06 \%$ & enfle-boeuf \\
\hline ferre & $\sigma$ & 1 & $0,06 \%$ & ferre-mule \\
\hline foule & $\sigma$ & 1 & $0,06 \%$ & foule-crapaud \\
\hline hume & $\sigma$ & 1 & $0,06 \%$ & hume-vent \\
\hline li & & 1 & $0,06 \%$ & licou \\
\hline mate & $\sigma$ & 1 & $0,06 \%$ & mate-faim \\
\hline prête & $\sigma$ & 1 & $0,06 \%$ & prête-nom \\
\hline
\end{tabular}

\begin{tabular}{|l|l|l|l|l|}
\hline amuse & $\sigma \sigma$ & 1 & $0,06 \%$ & amuse-gueule \\
\hline copie & $\sigma \sigma$ & 1 & $0,06 \%$ & copie-lettres \\
\hline écorche & $\sigma \sigma$ & 1 & $0,06 \%$ & écorche-cul \\
\hline épluche & $\sigma \sigma$ & 1 & $0,06 \%$ & épluche-légumes \\
\hline étouffe & $\sigma \sigma$ & 1 & $0,06 \%$ & étouffe-chrétien \\
\hline ratisse & $\sigma \sigma$ & 1 & $0,06 \%$ & ratisse-caisse \\
\hline rebrousse & $\sigma \sigma$ & 1 & $0,06 \%$ & rebrousse-chemin \\
\hline remonte & $\sigma \sigma$ & 1 & $0,06 \%$ & remonte-pente \\
\hline
\end{tabular}

Tableau 4. Verbes les moins fréquents dans les mots composés VN

Finalement, que l'on quantifie le nombre de verbes ou le nombre de mots composés, la préférence pour les radicaux monosyllabiques s'impose.

\subsubsection{Conséquences des contraintes de taille sur le choix des verbes}

Il y a donc tout lieu de penser que des contraintes de taille portent sur les verbes des mots composés VN et qu'elles influencent nettement la sélection des bases verbales. Ainsi, en admettant que les contraintes morphologiques ne jouent aucun rôle (puisque le corpus compte au moins un exemple de chaque type morphologique cf. ci-dessous), les contraintes de taille seraient responsables de l'évitement, par la composition VN de plusieurs groupes de verbes, aux radicaux trop longs, alors qu'ils répondent parfaitement bien aux attentes sémantiques de la règle. Seraient ainsi expliqués : 
(i) l'absence de verbes qui exèdent deux syllabes (cf. Tableau 1), alors qu'ils sont plus d'un tiers dans la liste des verbes du français (cf. Tableau 2). Ainsi, des verbes tri- ou quadri-syllabiques tels que domestiquer ou métamorphoser ne permettent de former aucun mot composé du corpus, quoiqu'ils soient sémantiquement conformes aux contraintes de la règle (on pourrait nommer un agent ${ }^{\circ}$ domestique-chien ou un instrument ${ }^{\circ}$ métamorphose-grenouille ${ }^{3}$ ).

(ii) la quasi absence de verbes construits par affixation, tels que les verbes préfixés (ex. 4), les verbes suffixés, incluant toute la suffixation évaluative (ex. 5) ou les verbes composés (ex. 6) :

adoucir, cohabiter, contre-manifester, décoloniser, échantillonner, ennoblir, préselectionner, postposer, réactualiser, sous-estimer, surprotéger, transparaître

diversifier, tranquilliser, écrivasser, discutailler, sautillonner, traficoter autofinancer, héliporter, hydrofuger, micro-injecter, photocopier

En effet, le corpus ne recense aucun verbe suffixé en -ifier, -iser ou même -oyer alors que les mots composés VN hypothétiques comme ${ }^{\circ}$ amplifie-son, ${ }^{\circ}$ purifie-eau, ${ }^{\circ}$ tranquillise-malade ou ${ }^{\circ}$ verdoiepelouse seraient sémantiquement conformes. De même, les bases verbales préfixées se révèlent extrêmement rares; seules apparaissent quelques bases préfixées à initiale vocalique (abaisse, abat, emporte) ou à initiale en /ra/ (relève, remonte, repose), c'est-à-dire celles dont l'initiale risque de ne pas former une bonne attaque. On dénombre par ailleurs un seul verbe construit en dé- (démonte-pneu).

(iii) l'absence quasi-totale de verbes du $2^{\text {ème }}$ groupe (le seul exemple est guérir dans guérit-tout, mais son statut de mot composé morphologique est contesté cf Fradin $2009^{4}$ ) : en effet, le radical sélectionné par la règle de composition correspondrait dans ce cas au radical court du verbe (à finale en /i/ pour les verbes $\mathrm{du} 2^{\text {ème }}$ groupe), et comporterait nécessairement deux syllabes ou plus. Par exemple, on pourrait imaginer des mots composés VN tels que ${ }^{\circ}$ nourrit-animal ${ }^{5}$ pour référer à un instrument qui déverse la nourriture aux animaux domestiques en l'absence de leurs maîtres, ${ }^{\circ}$ réussit-examen référant à un médicament ou gravit-montagne pour un sportif alpiniste. L'analyse vaut également pour l'absence d'autres radicaux verbaux non mono-vocaliques à finale en /i/, traditionnellement classés dans le $3^{\text {ème }}$ groupe (par exemple, on pourrait construire des mots composés $\mathrm{VN}$ tels que ${ }^{\circ}$ conduit-train, ${ }^{\circ}$ écrit-roman pour référer à un agent) $)^{6}$.

\subsection{Contraintes de taille sur les noms}

Cette section montre que les contraintes de taille sont moins rigoureuses sur les noms que sur les verbes, quoiqu'elles s'avèrent bien présentes.

\subsubsection{Des noms tendanciellement monosyllabiques}

L'étude de la taille des noms des mots composés VN du corpus a été menée en calculant le nombre de mots composés qui comprennent ces noms, plutôt qu'en comptabilisant le nombre de noms distincts figurant dans le corpus. En conséquence, un nom comme cigare qui apparaît dans plusieurs mots composés en combinaison avec plusieurs verbes (coupe-cigare, fume-cigare, porte-cigares), sera comptabilisé autant de fois que les mots composés qui le contiennent. Ce mode de calcul permet ainsi de fournir un aperçu plus réaliste des préférences des locuteurs.

Le Tableau 5 montre que le corpus comprend une forte majorité de mots composés VN construits avec un nom monosyllabique. Ainsi, sur les 1473 mots composés VN, 871 contiennent un nom monosyllabique, soit près de $60 \%$ (ex. 7) :

tournevis, sèche-linge, accroche-coeur 
Cette proportion est moins spectaculaire que celle qui concerne les verbes et montre que la règle offre une plus grande souplesse à l'égard de la taille des noms que de celle des verbes. En effet, un nombre non négligeable de noms dissyllabiques $(36,4 \%$ des composés $\mathrm{VN}$ ) figure dans les mots composés (ex. 8 )

aide-mémoire, brûle-parfum, ramasse-couverts

En revanche, les proportions chutent considérablement dès que l'on considère les noms de taille supérieure à deux syllabes : si les mots composés VN comprenant des noms trisyllabiques représentent encore $4 \%$ des mots composés totaux (ex. 9), la barre des 1\% n'est pas dépassée pour les mots composés avec un nom à quatre syllabes (ex. 10) ou cinq syllabes dont l'unique représentant est porte-épéebaïonnette. fume- $\underline{\text { cigarettes, }}$ monte-escalier, porte- $\underline{\text { allumettes }}$

$$
\text { lèse-humanité ; porte-hélicoptères, porte-isolateur }
$$

\begin{tabular}{lll}
\hline VN comprenant un & $\begin{array}{l}\text { Nombre } \\
\text { (total 1473) }\end{array}$ & Pourcentage \\
\hline - N monosyllabique & $\mathbf{8 7 1}$ & $\mathbf{5 9 \%}$ \\
- N dissyllabiques & $\mathbf{5 3 7}$ & $\mathbf{3 6 , 4} \%$ \\
- N trisyllabique & 59 & $4 \%$ \\
- N quadrisyllabique & 5 & $0,3 \%$ \\
- N à cinq syllabes & 1 & $0,07 \%$ \\
\hline
\end{tabular}

Tableau 5. Longueur des noms dans les mots composés VN

La préférence que la composition $\mathrm{VN}$ a pour les noms inférieurs à trois syllabes permet certainement d'expliquer la présence de mots composés comme presse-purée ${ }^{7}$ dont la sémantique du $\mathrm{N}$ ne répond pas aux propriétés prototypiques attendues par la règle : en effet, purée remplit ici le rôle sémantique de résultat au regard du verbe ('purée' est le résultat de 'presser') et non celui de patient comme la majorité des $\mathrm{N}$ des mots composés VN (ouvre-boîte). En somme, purée prend ici la place du nom pomme-de-terre sémantiquement proche, mais certainement trop long pour la formation ${ }^{8}$.

\subsubsection{Taille des noms dans le lexique général du français}

Ces résultats sont là encore à mettre en regard avec les proportions observées dans le lexique général du français. Sur les 28639 noms du français (thème singulier), 9\% seulement sont monosyllabiques tandis que $36 \%$ sont dissyllabiques et $32 \%$ sont trisyllabiques.

\begin{tabular}{lll}
\hline Thème 1 du nom : & Total (sur 28639 noms) & Pourcentage \\
\hline - monosyllabique & 2666 & $9 \%$ \\
- bisyllabique & $\mathbf{1 0 3 4 5}$ & $\mathbf{3 6 \%}$ \\
- trisyllabique & $\mathbf{9 3 7 0}$ & $\mathbf{3 2 \%}$ \\
- quatre syllabes & $\mathbf{4 3 1 2}$ & $\mathbf{1 5 \%}$ \\
- cinq syllabes & 1449 & $\mathbf{5 \%}$ \\
- six syllabes & 406 & $1,4 \%$
\end{tabular}

Tableau 6. Longueur des noms (thème singulier) dans le lexique général du français

Ces chiffres montrent qu'au contraire de ce qui est observé dans les mots composés VN, la taille des noms du français est très majoritairement supérieure à une syllabe : les noms les plus représentés comptent entre 2 et 4 syllabes, alors que les noms monosyllabiques, préférés par les composés VN, arrivent seulement en $4^{\text {ème }}$ position. La comparaison entre la taille des noms dans les mots composés VN 
et celle dans le lexique général du français conduit aux mêmes conclusions que celles avancées pour la taille des verbes : la forte représentation de noms monosyllabiques dans les mots composés VN n'est pas à l'image de la place qu'ils occupent dans le lexique général du français. En revanche, elle est la preuve que la règle de composition $\mathrm{VN}$ fait également peser des contraintes de taille sur les noms qu'elle sélectionne pour base.

Les chiffres sur le lexique général du français montrent en outre que comparativement aux verbes (ou plus précisément, au thème 3 des verbes), les noms (leur thème 1) sont potentiellement plus longs en français. En effet,

- d'une part, la longueur possible des noms est supérieure à celle des verbes : Lexique recense des noms comprenant jusqu'à 9 syllabes (sont cités, électroencéphalographie, oto-rhinolaryngologiste, hypercoagulabilité, qui au demeurant, seraient peu compatibles avec la composition VN), alors que la longeur maximale d'un thème verbal est de 5 syllabes (sont cités : universalise, emberlificote, réapprovisionne, occidentalise, intellectualise) ;

- d'autre part, les pourcentages révèlent une plus forte proportion de noms longs que de verbes longs : en effet, les verbes trisyllabiques représentent $23,8 \%$ des verbes alors que les noms de même longueur représentent $55 \%$ des noms ; quant aux verbes quadrisyllabiques, ils ne sont plus que $4,5 \%$ dans la base tandis que les noms de quatre syllabes représentent $23,2 \%$ des noms du corpus.

La différence de longueur potentielle entre noms et verbes dans le lexique général du français, associée à une contrainte de dénomination, pourrait contribuer à expliquer la raison pour laquelle la règle de composition autorise une plus grande souplesse à l'égard de la taille des noms qu'à l'égard de celle des verbes.

Cependant, la question qui reste en suspens est celle des objets sur lesquels portent les contraintes de taille : portent-elles davantage sur un des composants ou plutôt sur le construit dans son entier. La section qui suit tente de répondre à cette question.

\section{Mots composés VN : vers l'idéal disyllabique?}

La préférence que manifeste la règle de composition VN pour les verbes et les noms monosyllabiques, conduit-elle à former une majorité de mots composés dissyllabiques ? En d'autres termes, les contraintes de taille qui pèsent sur les composants seraient-elles la conséquence d'une contrainte contraignant les mots composés à répondre à un idéal dissyllabique ? Cette partie montre que, malgré tout, moins de la moitié des mots composés VN sont dissyllabiques. Il semble donc qu'il faille distinguer entre contrainte de taille qui porte sur les lexèmes en input de la règle et contrainte de taille en output.

\subsection{Des noms majoritairement monosyllabiques avec des verbes monosyllabiques}

Les noms associés aux verbes monosyllabiques sont majoritairement des monosyllabes. Ainsi, sur les 1348 noms qui suivent un verbe monosyllabique, 784 sont monosyllabiques, ce qui représente $58 \%$ des noms (à titre d'exemple, sou, mouche, plat, poche dans les mots composés en (11))

$$
\text { grippe-sou, tue-mouche, monte-plats, vide-poches }
$$

De même que les noms dissyllabiques sont très présents sur l'ensemble du corpus, ils le sont également en association avec des bases verbales monosyllabiques (37\%; ex. (12)), formant ainsi des mots composés trisyllabiques.

cache-lumière, vide-ordures, saute-mouton 
Comme attendu, la règle $\mathrm{VN}$ autorise également l'association de verbes mono-syllabiques avec des noms comprenant plus de deux voyelles, puisque l'on comptabilise 56 noms trisyllabiques (4,15\%) (ex. 13), ainsi que les 5 noms à quatre voyelles et l'unique nom de cinq voyelles déjà recensés dans le corpus global, formant ainsi des mots composés pouvant compter jusqu'à six syllabes.

$$
\text { fume-cigarettes, monte-escalier, porte- } \underline{\text { allumettes }}
$$

\subsection{Recalcul syllabique des mots composés di- et tri-syllabiques}

Il semblerait à première vue que, malgré la capacité certaine qu'a la règle d'accueillir des noms plurisyllabiques, les chiffres confortent le sentiment qu'un mot composé VN tend à répondre à l'idéal dissyllabique des mots du français : en ramenant les décomptes à l'ensemble du corpus (1473 mots composés $\mathrm{VN}$ ), et en additionnant le nombre de $\mathrm{VN}$ où un verbe monosyllabique est associé à un nom monosyllabique (784), on obtiendrait $53 \%$ de mots composés VN dissyllabiques.

Mais ce serait sans compter les bases verbales monosyllabiques dont la finale est composée de deux consonnes suivies d'un schwa telles que ouvre, porte, tourne et qui, suivies d'un nom monosyllabique à initiale consonantique (boîte, plume, disque), imposent la réalisation du schwa (cf. § 3.1.1), formant ainsi des verbes dissyllabiques dans des mots composés trisyllabiques (ouvre-boîte, porte-plume, tournedisque). Or ces cas sont nombreux et il arrive même que pour un même verbe, ils représentent presque la moitié. Au final, 232 mots composés VN dont le décompte syllabique initial recensait un verbe monosyllabique suivi d'un nom monosyllabique, doivent être comptabilisés comme trisyllabiques, le verbe devant être analysé comme un dissyllabe suite à la réalisation du schwa final. Les mots composés en (14), ajoutés à ceux cités ci-dessus, en sont autant d'exemples

ferme-bourse, garde-robe, porte-ski, cherche-fuite, couvre-lit, perce-neige, trouble-fête.

Ce décompte réduit immanquablement le nombre de mots composés dissyllabiques qui ne sont plus que 552 sur un ensemble de 1473 et ne représentent plus que $37 \%$ des mots composés totaux.

Ce pourcentage peut néanmoins être revu à la hausse en examinant plus attentivement les mots composés VN trisyllabiques et en y intégrant ceux dont une des syllabes pourrait être exclue du décompte syllabique à cause d'une mauvaise attaque de syllabe initiale ou d'une syllabe tombant suite à la chute d'un schwa. Cette situation concerne principalement les verbes dans la mesure où (i) l'initiale vocalique des noms ne peut généralement pas être exclue du décompte syllabique car elle forme une syllabe avec la consonne finale du verbe qui précède (ex. 15), (ii) le premier schwa du nom ne tombe pas si le verbe se termine par une consonne (ex. 16) (Dell $1973: 225-226)$.

$$
\text { cache-entrée, chasse-ennui, coupe-oreille, monte- } \underline{\text { acide, }} \text {, pèse-alcool }
$$

chauffe-chemise, coupe-cheville, monte-ressort, trompe-cheval

Mais parmi les 318 mots composés VN trisyllabiques comprenant des verbes dissyllabiques associés à des noms monosyllabiques, 44 sont construits de verbes à initiale vocalique (ex. 17) et 10 de verbes à initiale consonantique en /rə/ (ex. 18), autant de verbes dont la syllabe initiale pourrait être exclue du décompte syllabique, les ramenant ainsi à des monosyllabes (cf. Tableau 7).

$$
\text { abaisse-langue, attrape-mouche, essuie-glace }
$$

remonte-pente, remue-ménage, repose-bras

En conséquence, 54 noms composés VN (verbe dissyllabique et nom monosyllabique) sur les 318 initialement comptabilisés comme trisyllabiques peuvent être ajoutés aux mots composés dissyllabiques. 


\begin{tabular}{llll}
\hline $\begin{array}{l}\text { V disyllabique d'un composé } \\
\text { comportant: }\end{array}$ & $\begin{array}{l}\text { Nombre de V } \\
\text { (total 31) }\end{array}$ & $\begin{array}{l}\text { Nombre de } \\
\text { trisyllabiques } \\
\text { (total 318) }\end{array}$ & $\begin{array}{l}\text { VN Pourcentage } \\
\text { trisyllabiques }\end{array}$ \\
\hline $\begin{array}{l}\text { - deux voyelles pleines dont une à } \\
\text { l'initiale }\end{array}$ & 44 & $14 \%$ \\
- deux voyelles dont initiale en /rə/ & 5 & 10 & $3 \%$ \\
- deux voyelles sans initiale V ni 7 & 232 & $73 \%$ \\
/ro/ & & \\
\hline
\end{tabular}

Tableau 7. Verbes dissyllabiques dans des composés trisyllabiques

$\mathrm{Au}$ final, sur les 1473 mots composés VN du corpus 606 sont analysables comme dissyllabiques (comprenant 552 dissyllabes et 54 trisyllabes recalculés), si tant est que l'on valide ces nouveaux calculs. Le nombre de mots composés VN dissyllabiques représente ainsi au maximum $41 \%$ de l'ensemble. Ces chiffres montrent que la mise en conformité du mot composé VN à un schème dissyllabique n'est pas une priorité. Cet idéal dissyllabique représente uniquement la taille minimale que doit avoir un mot composé VN. La question se pose dorénavant de la taille maximale optimale qu'il aurait. En d'autres termes, des contraintes de taille ne joueraient-elles pas sur le mot composé lui-même? La partie 5 a pour objet d'évaluer la plausibilité de cette hypothèse.

\section{Des contraintes de taille dans les composés pluri-syllabiques}

Une étude quantitative plus détaillée de la longueur des noms relativement à la longueur des verbes tend à confirmer que les contraintes de taille dans les mots composés VN touchent aussi le mot composé dans son ensemble. Cette section présente des arguments allant dans ce sens : nous montrerons que bien que les mots composés VN puissent excéder deux syllabes, c'est-à-dire dépasser la taille idéale du mot du français, la tendance est néanmoins à privilégier les mots composés les plus courts possibles. Cette tendance est visible dans le choix de la longueur du nom et du verbe relativement à la longueur respective de l'autre composant. Ainsi, les données révèlent que les verbes longs s'associent très préférentiellement à des noms courts ( $§ 5.1)$, et parallèlement, que les noms longs s'associent à des verbes courts ( $§ 5.2)$.

\subsection{Majorité de $\mathbf{N}$ courts avec des $\mathbf{V}$ longs}

L'étude de la taille des noms associés à des verbes longs (c'est-à-dire, dans notre corpus, à des verbes dissyllabiques) montre qu'ils sont en très nette majorité mono-syllabiques (cf. Tableau 8). Un premier décompte montre que, parmi les 356 noms du corpus qui s'associent à un verbe dissyllabique, quasiment 90\% sont monosyllabiques (ex. 19) et seulement $10 \%$ environ dissyllabiques (ex. 20).

arrache-clou, repose-pieds, copie-lettres

essuie-voitures, allume-cigare, protège-cahier

La proportion de noms supérieurs à trois syllabes est encore plus éloquente : tandis que $0,8 \%$ des noms qui peuvent se combiner avec des verbes dissyllabiques sont trisyllabiques (ex. 21), plus aucun nom de 4 ou 5 syllabes n'est recensé.

protège-radiateur, protège-objectif, protège-parapluie 
Ces chiffres confirment la propension de la règle à privilégier la construction de mots composés les plus courts possibles.

\begin{tabular}{lll}
\hline $\mathrm{N}$ associés à un V dissyllabique & Nombre (355 noms) & Pourcentage \\
\hline$-\mathrm{N}$ monosyllabiques & 318 & $89,5 \%$ \\
$-\mathrm{N}$ dissyllabiques & 34 & $9,6 \%$ \\
$-\mathrm{N}$ trisyllabiques & 3 & $0,8 \%$ \\
$-\mathrm{N}$ quadrisyllabiques & 0 & $0 \%$ \\
$-\mathrm{N}$ à cinq syllabes & 0 & $0 \%$ \\
\hline
\end{tabular}

Tableau 8. Longueur des noms combinés à des verbes dissyllabiques

Un second décompte prenant en compte la qualité des attaques des verbes et noms dissyllabiques fournirait des chiffres encore plus radicaux. Sans entrer dans les détails, nous pouvons néanmoins mentionner à titre illustratif que, sur les 34 mots composés VN quadrisyllabiques construits avec des verbes et des noms dissyllabiques, seuls 5 le sont assurément, ceux construits avec la base verbale protège (ex. 22).

$$
\text { protège-cabine, protège-cahier, protège-genou, protège-oreilles, protège-tibia }
$$

Les autres VN sont construits de verbes a priori dissyllabiques réanalysables comme monosyllabiques à cause d'une syllabe initiale comprenant une mauvaise attaque, en /rə/ (ex. 23) ou vocalique (ex. 24).

$$
\begin{aligned}
& \text { relève-moustache, remonte-pente, remue-ménage, repose-bras } \\
& \text { essuie-voitures, abaisse-paupière, enfile-aiguilles, épluche-légumes }
\end{aligned}
$$

\subsection{Majorité de $\mathrm{V}$ courts avec des $\mathrm{N}$ longs}

L'étude de la taille des verbes associés à des noms longs (de plus de deux voyelles) montre que, plus les noms comptent un nombre important de syllabes, plus ils s'associent à des verbes courts, monosyllabiques. En effet, aucun nom comportant quatre syllabes (humanité, aéronefs, hélicoptères, isolateur, baïonnette) ou cinq (épée-baïonnette) ne s'associe avec un verbe long; ils se combinent systématiquement avec des verbes monosyllabiques (ex. 25). Les noms trisyllabiques ne sont que 3 à s'associer à un verbe long (ex. 26) et les noms dissyllabiques sont uniquement 34 à s'associer à un verbe long.

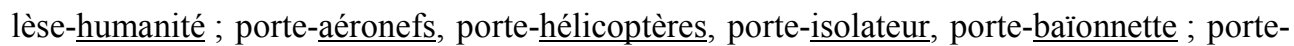
épée-baïonnette

protège-objectif, protège-parapluie, protège-radiateur

Ces résultats conduisent à conclure qu'en dépit du fait que certains noms soient pluri-syllabiques, formant ainsi des mots composés dont la longueur dépasse l'idéal dissyllabique du mot optimal du français, la règle tend systématiquement vers la moindre longueur du composé.

\section{Conclusion}

L'analyse quantitative des données du corpus du point de vue de la taille des lexèmes en jeu montre clairement que

(i) les verbes des mots composés $\mathrm{VN}$ du français sont soumis à des contraintes de taille et doivent répondre, à la différence des verbes du français en général, au format d'un monosyllabe ; 
(ii) les noms des mots composés VN subissent également des contraintes de taille (ils n'atteignent jamais dans un mot composé VN la taille des noms du français), contraintes moins lourdes cependant que pour les verbes puisqu'ils parviennent à s'imposer plus facilement avec une taille supérieure ;

(iii) la majorité des mots composés $\mathrm{VN}$ ne se moule pas dans le format optimal d'un mot du français puisque moins de la moitié d'entre eux est dissyllabique. Des contraintes de taille s'imposent pour autant avec force sur le mot composé pour tendre vers cet idéal : plus de 50\% comptent trois syllabes et très rares sont ceux qui dépassent ce format, (cf. Tableau 9).

\begin{tabular}{lll}
\hline Taille du VN & $\begin{array}{l}\text { Nombre } \\
\text { (total 1473) }\end{array}$ & Pourcentage \\
\hline - VN dissyllabiques & $\mathbf{6 0 6}$ & $\mathbf{4 1} \%$ \\
- VN trisyllabiques & $\mathbf{8 0 2}$ & $\mathbf{5 4 , 4 \%}$ \\
- VN quadrisyllabiques & 59 & $4 \%$ \\
- VN à cinq syllabes & 5 & $0,3 \%$ \\
- VN à six syllabes & 1 & $0,06 \%$ \\
\hline
\end{tabular}

Tableau 9. Longueur des mots composés VN du français

Ces chiffres révèlent que la taille optimale des mots composés $\mathrm{VN}$ du français est entre deux et trois syllabes. Les rares mots composés de taille supérieure répondent de toute évidence à un besoin énonciatif qui supplante alors la containte de taille (la nécessité de fournir une dénomination précise l'emporte alors sur la longueur).

Cependant, bien que des contraintes de taille portent sur le mot composé dans son entier, privilégiant les combinaisons verbe-nom les plus brèves possibles, ces contraintes jouent principalement sur la base verbale. Une hiérarchie semble ainsi exister entre contrainte de taille sur le verbe et contrainte de taille sur le nom.

Ces résultats constituent des preuves empiriques supplémentaires aux observations de Lignon, Plénat et Roché sur d'autres données du français (taille minimale de deux syllabes observée notamment sur la prononciation des sigles et les suffixations substitutives, taille maximale entre deux et trois syllabes dans les troncations et suffixation décallée, contrainte de taille ne portant que sur le radical dans les suffixations en -issime). La mise au jour de ces contraintes a aussi valeur explicative puisqu'elle justifie l'absence de certains verbes dans le corpus de composés $\mathrm{VN}$, qui pourtant répondent bien aux propriétés sémantiques attendues: principalement les verbes construits par affixation (suffixés, préfixés) et les verbes du $2^{\text {ème }}$ groupe.

Il reste encore à mesurer le poids que représentent les contraintes de taille face à d'autres contraintes concurrentes en jeu dans la règle de composition VN. Par exemple, la contrainte de dénotation d'une entité semble pouvoir violer, dans une certaine mesure, la contrainte la taille (un mot composé VN peut compter 5 syllabes s'il est besoin de dénoter ce qu'il doit dénoter). En revanche, la contrainte sémantique qui pèse sur le verbe ne semble pas pouvoir triompher des contraintes de taille (cf. l'éviction des verbes du $2^{\text {ème }}$ groupe parfaitement conformes sémantiquement). Quant à la concurrence entre contrainte phonologique de taille et contrainte morphologique, le corpus de mots composés VN étudié fournit une réponse claire : la contrainte morphologique de sélection du thème 3 l'emporte sur les contraintes de taille. On aurait pu penser que cette contrainte morphologique était motivée par les contraintes phonologiques de taille : pour la majorité des verbes des mots composés $\mathrm{VN}$, le thème 3 est celui que le verbe ait de plus court. Cela est vrai, à l'exclusion des verbes du $2^{\text {eme }}$ groupe dont le thème le plus court est le thème zéro. En effet, pour ces verbes, le thème verbal optimal pour répondre aux exigences de taille aurait été le thème zéro. Le thème zéro est défini par Tribout (2010) qui, à l'occasion de l'étude des converts $\mathrm{V}>\mathrm{N}$, a formulé l'hypothèse de l'existence d'un radical supplémentaire des verbes par rapport à ce que proposait Bonami \& Boyé (2003). Le « thème zéro », sélectionné par la conversion déverbale mais invisible à la flexion correspond pour les verbes du $2^{\text {ème }}$ groupe, au thème 3 (le thème court) moins le /i/ final. Ainsi, le radical choisi par la conversion $\mathrm{V}>\mathrm{N}$ n'est pas celui qui laisse apparaître le /i/ final habituellement enregistré au titre du radical, mais celui-ci moins le /i/ : en effet, le convert construit sur la 
base du verbe bondir n'est pas ${ }^{\circ}$ bondi mais bond; celui sur la base du verbe ressortir n'est pas ${ }^{\circ}$ ressorti mais ressort, celui sur enchérir est enchère et celui sur meurtrir est meurtre. En revanche, le thème zéro de tous les autres verbes est identique au thème 3 du verbe.

Ainsi, la composition $\mathrm{VN}$, en sélectionnant le thème zéro des verbes, aurait eu les moyens morphologiques de répondre aux exigences phonologiques des contraintes de taille pour les verbes du 2ème groupe. Mais la sélection du thème 3 du verbe, dans ce cas trop long, force à constater la supériorité des contraintes morphologiques sur les contraintes phonologiques de taille dans l'échelle des hiérarchies des contraintes de formation des composés VN.

Ces résultats ouvrent de nouvelles perspectives de recherche, tant d'un point de vue empirique que théorique.

Pour ce qui est des données, l'examen d'autres règles constructionnelles peut venir illustrer la supériorité des contraintes morphologiques sur les contraintes phonologiques de taille. Notre attention se tourne en premier lieu vers les converts nominaux à base verbale : outre le fait qu'ils partagent avec les mots composés VN nombre de leurs propriétés sémantiques, catégorielles et l'absence de marque affixale sur le radical (apparition du radical nu du verbe : sauter $>$ saut, marcher $>$ marche, nager $>$ nage), ils n'admettent également que très peu de bases verbales construites déverbales (aucun convert sur base suffixée en -iser et -ifier n'est recensé hormis un exemple), et, le cas échéant, ces bases ne dépassent pas deux syllabes (Tribout 2010). La conversion $\mathrm{V}>\mathrm{N}$ semble ainsi offrir un nouvel exemple de procédé morphologique où des contraintes de taille sont à l'œuvre mais supplantées par des contraintes morphologiques : en effet, la conversion, en sélectionnant le thème zéro du verbe (cf. exemples cidessus), fournit des converts monosyllabiques, inférieurs à la taille optimale du mot français.

Pour ce qui concerne les questions théoriques, l'étude des mots composés VN conduit à s'interroger sur la concurrence entre contraintes de divers plans au sein d'une même règle morphologique : comment les contraintes morphologiques viennent-elles interférer avec les contraintes sémantiques, et comment entrent-elles en concurrence avec les contraintes phonologiques. A quelles conditions l'une vient l'emporter sur l'autre?

Par ailleurs, se pose la question de la représentation du poids respectif de ces contraintes et de leur hiérarchie dans la formulation de la règle. Par exemple, on doit pouvoir représenter au titre des propriétés phonologiques de la règle de composition $\mathrm{VN}$, l'impact qu'exercent les contraintes de taille sur le verbe, le nom et le mot composé lui-même et leur poids les unes relativement aux autres. De même faudrait-il que la règle puisse rendre compte de la hiérarchie des contraines phonologiques, morphologiques et sémantiques entre elles. C'est-à-dire, dans le cas des mots composés VN, donner à voir que la contrainte morphologique supplante la contrainte de taille dans le choix du verbe, (la sélection du thème est stable) mais pour autant la contrainte phonologique écrase, à une certaine étape de la construction la contrainte sémantique en évinçant quantité de verbes du $2^{\text {ème }}$ groupe.

\section{Références bibliographiques}

Anderson, S. R. (1992). A-Morphous Morphology. Cambridge : Cambridge University Press.

Aronoff, M. (1994). Morphology by itself. Cambridge : MIT Press.

Bonami O. \& Boyé G. (2003). Supplétion et classes flexionnelles. Langages 152, 103-126.

Bonami O. \& Boyé G. (2005). Construire le paradigme d'un adjectif. Recherches linguistiques de Vincennes 34, 7798.

Bonami, O. Boyé, G., Kerleroux, F. (2009). L’allomorphie radicale et la relation flexion-construction. Fradin B., Kerleroux F. \& Plénat M. (eds). Aperçus de morphologie du français. Saint-Denis: Presses Universitaires de Vincennes, 103-125.

Corbin, D. (1992). Hypothèses sur les frontières de la composition nominale. Cahiers de grammaire 17, 26-55.

Corbin, D. (à paraître). Le lexique construit. Paris : Armand Colin.

Dell, F. (1973). Les règles et les sons. Introduction à la phonologie generative. Paris : Hermann.

Fradin, B. (2003). Nouvelles approches en morphologie. Paris : Puf.

Fradin, B. (2009). Compounding in French. Lieber R. and P. Štekauer (eds). Oxford Handbook on Compounding. Oxford : Oxford University Press, 417-435. 
Kilani-Schoch, M. \& Dressler, W. U. (1992). Prol-o, intell-o, gauch-o et les autres. Propriétés formelles de deux opérations du français parlé. Romanistisches Jahrbuch, 65-86.

Lignon, S. (1999). Suffixasser ou suffixouiller ?. Silexicales $n^{\circ} 2$, La morphologie des dérivés évaluatifs, Actes du colloque de Toulouse, 29-30 avril 1999, 117-126.

Lignon, S. (à paraître). -iser and -ifier suffixations in French : Checkify data to checkize hypotheses ?. N. Hathout \& F. Montermini (eds). Papers from the 7th Décembrettes. München : Lincom Europa.

New B., Pallier C., Ferrand L., Matos R. (2001). Une base de données lexicales du français contemporain sur internet: LEXIQUE. L'Année Psychologique 101, 447-462. http://www.lexique.org.

Plénat, M. (1993). Observations sur le mot minimal français. Laks B. \& Plénat M. (eds). De natura sonorum. SaintDenis: Presses universitaires de Vincennes, 143-172.

Plénat M.. (1984). Toto, Fanfa, Totor et même Guiguitte sont des ANARS. Dell F., Hirst D. \& Vergnaud J.-R. (eds).Forme sonore du langage Structure des représentations en phonologie. Paris: Hermann, 161-181.

Plénat, M. (1997a). Morphophonologie des dérivés en -Vche. Recherches linguistiques de Vincennes 26. Saint-Denis, Presses universitaires de Vincennes, 113-150.

Plénat, M. (1997b). De quelques paramètres intervenant dans l'oralisation des sigles en français. Cahiers d'Etudes Romanes (CERCLID) 9, 27-52.

Plénat, M. (1999). Prolégomènes à une étude variationniste des hypocoristiques à redoublement en français. Cahiers de grammaire $24,183-219$.

Plénat, M. (2000). Quelques thèmes de recherche actuels en morphophonologie française. Cahiers de lexicologie 77, $27-62$.

Plénat, M. (2002). Jean-Louis Fossat : fossatissime. Note sur la morphophonologie des dérivés en -issime. Hommage à Jean-Louis Fossat [= Cahiers d'Etudes Romanes (CERCLID) 11/12]. Rabassa L. (éd.). Toulouse : Université Toulouse 2, CLID, 229-248.

Plénat, M. (2005). Rosinette, cousinette, starlinette, chipinette. Décalage, infixation et épenthèse devant -ette. Questions de classification en linguistique : méthodes et descriptions. Choï-Jonin I., Bras M., Dagnac A. \&. Rouquier M (éds). Mélanges offerts au Professeur Christian Molinier. Berne, :Peter Lang, 275-298.

Plénat, M. (2008). Le thème L de l'adjectif et du nom. Durand J., Habert B.et Laks B. (éds). Actes du Congrès Mondial de Linguistique Française 2008. Paris : Institut de Linguistique Française, 1613-1626.

Plénat, M., (2009). Les contraintes de taille. Fradin B., Kerleroux F. \& Plénat M. (éds). Aperçus de morphologie du français. Saint-Denis : Presses Universitaires de Vincennes, 47-64.

Plénat, M., \& Roché, M. (2003). Prosodic constraints on suffixation in French. Booij G., DeCesaris J., Ralli A. \& Scalise S. (eds). Topics in Morphology. Selected Papers from the Third Mediterranean Morphology Meeting (Barcelona, September 20-22, 2001). Barcelona, I.U.L.A. :Universitat Pompeu Fabra, 285-299.

Plénat, M. \& Roché, M. (2004). Entre morphologie et phonologie : la suffixation décalée. Lexique 16, 159-198.

Prince A. \& Smolensky P. (1993). Optimality Theory: Constraint Interaction in Generative Grammar. Rutgers : University Center for Cognitive Science Technical Report 2.

Roché, M. (1997). Briard, bougeoir et camionneur : dérivés aberrants, dérivés possibles. Corbin D., Fradin B., Habert B., Kerleroux F. \& Plénat M. (éds). Mots possibles et mots existants, Forum de morphologie (lères rencontres), Silexicales 1 Villeneuve d'Ascq, 241-250.

Roché, M. (2002). La suffixation décalée avec oc. -ièr(a). Rabassa L. (éd.) Cahiers d'Etudes Romanes 11-12. Mélanges offerts à Jean-Louis Fossat, 319-334.

Roché, M. (2003). L'interfixe est-il une unité morphologique ? Fradin B., Dal G., Hathout N., Kerleroux F., Plénat M. \& Roché M. (éds.). Les unités morphologiques, Actes du 3e Forum International de Morphologie (Villeneuve d'Ascq, 19-21 septembre 2002), Silexicales 3, 169-178.

Roché, M. (2009). Un ou deux suffixes ? Une ou deux suffixations ?. Fradin B., Kerleroux F. \& Plénat M. (éds). Aperçus de morphologie du français. Saint-Denis : Presses Universitaires de Vincennes, 143-173.

Roché, M. (2010). Base, thème, radical. Recherches Linguistiques de Vincennes 39, 95-133.

Tribout, D. (2010). Les conversions de nom à verbe et de verbe à nom en français. Thèse de doctorat, Université Paris-Diderot-Paris 7.

Villoing, F. (2002). Les mots composés $[V N]_{N / A}$ du français : réflexions épistémologiques et propositions d'analyse. Mémoire de Thèse de doctorat, Université Paris X-Nanterre.

Villoing, F. (2009). Les mots composés VN. Fradin B., Kerleroux F. \& Plénat M. (éds). Aperçus de morphologie du français. Saint-Denis : Presses Universitaires de Vincennes, 175-198.

Villoing, F. (2012). French compounding. Probus, International Journal of Latin and Romance Linguistics. Mouton de Gruyter.

\footnotetext{
${ }^{1}$ Je tiens à remercier avec chaleur Stéphanie Lignon, Françoise Kerleroux, Marc Plénat, Michel Roché et deux
} relecteurs anonymes pour leurs commentaires à une version antérieure de cet article, pour les idées que nous avons 
échangées et pour les petits secrets des méthodes d'analyse morphophonologiques qu'ils ont partagées avec moi avec beaucoup de générosité.

${ }^{2}$ Un corpus lexicographique pose nécessairement le problème de l'hétérogènéité en termes de fréquence et de disponibilité dans le lexique; il faudrait l'augmenter d'un corpus plus contemporain relevé dans des corpus électroniques, voire sur la Toile, et d'une étude de la fréquence des termes afin d'évaluer quels sont les mots composés les plus courants.

${ }^{3}$ L'emploi de la pastille est réservé aux exemples inventés par l'auteur et non attestés.

${ }^{4}$ Fradin (2009), en suivant les critères de Corbin (1992) qui permettent de répartir les mots composés dans les deux modules de la grammaire, analyse guérit-tout comme une construction syntaxique lexicalisée.

${ }^{5}$ Nous prenons le parti d'orthographier le verbe tel qu'il apparait en flexion à l'indicatif présent singulier de sorte à l'harmoniser avec d'autres formes verbales de mots composés attestés tels que cuit-œuf ou essuie-main, qui s'alignent sur la forme fléchie correspondante.

${ }^{6}$ Remarquons néanmoins que la finale en /i/ de radicaux verbaux supérieurs à une syllabe n'est pas impossible pour les verbes du premier groupe : le corpus fournit plusieurs exemples de mots composés comprenant les verbes appuyer (appuie-bras, appuie-livres, appuie-tête), copier (copie-lettres), et essuyer (essuie-glace, essuie-pieds, essuie-verres). Les radicaux verbaux à initiale vocalique pouvant être comptabilisés comme mono-vocaliques plutôt que bivocaliques à cause de la potentielle mauvaise attaque de la première syllabe; les difficultés à les exploiter dans un mot composé VN ne se poseraient alors pas. La possibilité d'avoir un radical bivocalique à finale en /i/ dans un mot composé VN se réduirait ainsi à l'unique exemple du mot composé copie-lettres parmi les 1473 mots composés VN. Il est fort probable que dans ce cas, la nécessité de dénotation l'emporte sur la contrainte de taille.

${ }^{7}$ Merci à Marc Plénat pour m'avoir soufflé cet exemple.

${ }^{8}$ Notons cependant, suite à une remarque d'un relecteur anonyme, que certains sites marchands de la Toile vendent des presse pomme de terre ou presse pommes de terre à côté des presse-purée. Mais rien ne prouve que cette unité soit adoptée par les locuteurs ; à l'heure actuelle, aucun dictionnaire ne la recense. 